\title{
Use of Streptococcus salivarius KI 2 in the prevention of streptococcal and viral pharyngotonsillitis in children
}

This article was published in the following Dove Press journal:

Drug, Healthcare and Patient Safety

13 February 2014

Number of times this article has been viewed

\author{
Francesco Di Pierro' \\ Maria Colombo ${ }^{2}$ \\ Alberto Zanvit ${ }^{3}$ \\ Paolo Risso ${ }^{4}$ \\ Amilcare S Rottoli ${ }^{5}$ \\ 'Scientific Department, Velleja \\ Research, Milan, ${ }^{2}$ Pediatric \\ Department, University of Parma, \\ Parma, ${ }^{3}$ Stomatology Institute, \\ Milan, ${ }^{4}$ Laboratory of Epidemiology \\ and Social Psychiatry, Mario \\ Negri Institute, Milan, ${ }^{5}$ Pediatric \\ Department, Uboldo Hospital, \\ Cernusco sul Naviglio, Italy
}

Background: Streptococcus salivarius K12 is an oral probiotic strain releasing two lantibiotics (salivaricin A2 and salivaricin B) that antagonize the growth of S. pyogenes, the most important bacterial cause of pharyngeal infections in humans also affected by episodes of acute otitis media. S. salivarius K12 successfully colonizes the oral cavity, and is endowed with an excellent safety profile. We tested its preventive role in reducing the incidence of both streptococcal and viral pharyngitis and/or tonsillitis in children.

Materials and methods: We enrolled 61 children with a diagnosis of recurrent oral streptococcal disorders. Thirty-one of them were enrolled to be treated daily for 90 days with a slow-release tablet for oral use, containing no less than 1 billion colony-forming units/tablet of S. salivarius K12 (Bactoblis ${ }^{\circledR}$ ), and the remaining 30 served as the untreated control group. During treatment, they were all examined for streptococcal infection. Twenty children (ten per group) were also assessed in terms of viral infection. Secondary end points in both groups were the number of days under antibiotic and antipyretic therapy and the number of days off school (children) and off work (parents).

Results: The 30 children who completed the 90-day trial with Bactoblis ${ }^{\circledR}$ showed a significant reduction in their episodes of streptococcal pharyngeal infection $(>90 \%)$, as calculated by comparing the infection rates of the previous year. No difference was observed in the control group. The treated group showed a significant decrease in the incidence $(80 \%)$ of oral viral infections. Again, there was no difference in the control group. With regard to secondary end points, the number of days under antibiotic treatment of the treated and control groups were 30 and 900 respectively, days under antipyretic treatment 16 and 228, days of absence from school 16 and 228, and days of absence from work 16 and 228. The product was well tolerated by the subjects, with no side effects, and only one individual reported bad product palatability and dropped out.

Conclusion: Prophylactic administration of S. salivarius K12 to children with a history of recurrent oral streptococcal disease resulted in a considerable reduction of episodes of both streptococcal and viral infections and reduced the number of days under antibiotic and/or antipyretic therapy and days of absence from school or work.

Keywords: Blis K12, pediatric trial, Bactoblis, S. pyogenes, antibiotic therapy

\section{Introduction}

Streptococcus salivarius K12 is a strain isolated from the throat of a New Zealand child, ${ }^{1}$ and capable of producing two distinct lantibiotics - salivaricin A2 and salivaricin B - encoded on two adjacent loci on a 190-kilobase megaplasmid. ${ }^{2}$ Owing to these two salivaricins, the K12 strain successfully counteracts the growth of $S$. pyogenes in vitro. ${ }^{3}$ In contrast the $\mathrm{K} 12 \mathrm{P}^{-}$strain (negative plasmid) is totally ineffective. ${ }^{4}$
Correspondence: Francesco Di Pierro Scientific Department, Velleja Research, 23 Viale Lunigiana, Milan 20I25, Italy Tel +393495527663

Fax +390523 5I I 894

Email f.dipierro@vellejaresearch.com 
The K12 strain is not only effective against $S$. pyogenes but also inhibits the growth of such pathogens as Haemophilus influenzae, S. pneumoniae, and Moraxella catarrhalis, all of which are involved in the etiopathogenesis of acute otitis media. ${ }^{5}$ The four pathogens are responsible for almost all bacterial pharyngotonsillitis cases in children and adults. ${ }^{6}$ The results of K12 administration in children demonstrate that the strain can colonize the oral cavity in some $30 \%$ of children as early as on the third administration day, with clear colonization even in the nasopharynx and adenoids, ${ }^{7}$ and can remain in tissue for up to 32 days after the last administration. ${ }^{8}$ The K12 strain is also endowed with an excellent antibiotic-sensitivity profile and high safety characteristics, as demonstrated in tests in animals ${ }^{9}$ and humans. ${ }^{10}$ Recent clinical trials, conducted both in adults ${ }^{11}$ and children, ${ }^{12}$ demonstrated that treatment with the K12 strain reduces recurrences of bacterial pharyngotonsillitis by approximately $80 \%$ and $90 \%$, respectively. Even if a treatment over at least 90 days is followed by a 6-month washout period, the protection rates against recurrence remain high (about $60 \%$ in either case). Preliminary data indicate that treatment with the K12 strain also seems to reduce acute otitis media recurrences by $40 \%$ in children. ${ }^{12}$ As the K12 strain can also inhibit proliferation of Micrococcus luteus, S. anginosus, Eubacterium saburreum, and Micromonas micros, it has been successfully used in the treatment of halitosis by eliminating volatile sulfur compounds in 14 days. ${ }^{13,14} \mathrm{~A}$ recent study highlighted the action of the K12 strain against Candida albicans, though in this case the authors did not demonstrate a cytotoxic action referable to the release of the two salivaricins (A2 and B), but reported a mechanical antihyphae action. ${ }^{15}$ In addition to the aforementioned biological actions, the K12 strain also seems to possess preventive properties against oropharyngeal infections of viral origin and gingivitis. This possibility seems to be confirmed by the fact that administration of the K12 strain in adults may increase salivary interferon- $\gamma$ levels without modifying the levels of either interleukin (IL)-1 $\beta$ or tumor necrosis factor- $\alpha$, but considerably reducing IL- 8 release. ${ }^{5}$ The incidence of pharyngotonsillitis is very high in children, and is caused by bacteria in about $35 \%$ of cases (with $80 \%$ of these being caused by $S$. pyogenes) and by respiratory viruses in about $65 \%$ of cases. ${ }^{16,17}$ We resolved to verify the prophylactic action of the K12 strain administered in children with a diagnosis of recurrent pharyngotonsillitis caused by $\beta$-hemolytic streptococcus, while assessing the aspects of potential prevention of either $S$. pyogenes infections or possible viral infections.

\section{Materials and methods}

\section{The product}

The K12 strain was formulated in the form of slowly dissolving oral tablets by SIIT (Trezzano, Milan, Italy) and notified to the Italian Ministry of Health as Bactoblis ${ }^{\circledR}$ by Omeopiacenza (Pontenure, Italy), according to the provisions of law 169 of 2004, on July 5, 2011 (notification number 53435). The preparation of Bactoblis ${ }^{\circledR}$ used in the clinical trial contained no less than 1 billion colony-forming units (CFU)/ tablet of S. salivarius K12 (BLIS Technologies, Dunedin, New Zealand).

\section{The clinical trial}

The multicenter, open, nonrandomized, controlled clinical trial was conducted on 61 pediatric individuals enrolled in the area of Milan, Italy, and treated between January 31 and April 30, 2013. The trial population consisted of 32 males (53.4\%) and 29 females (46.6\%). The trial was conducted according to the criteria set by the Declaration of Helsinki and with the approval of the local ethics committee. The parents of all the participants in the study were informed of the trial methods and signed the consent and privacy-policy documents.

\section{Inclusion criteria}

All the enrolled individuals were 3-13 years of age and attended school in the Milan area. In terms of recurrent pharyngotonsillitis, the enrolled individuals exhibited an average of not less than three episodes in the same quarter as that of the study (January 31 to April 30) of the previous year (2012). The episodes were confirmed by a rapid swab positive for group A and group B streptococci. None of the individuals were affected by infectious diseases of any nature on enrolment.

\section{Exclusion criteria}

The following exclusion criteria were used: immunocompromised individuals, individuals who had undergone tonsillectomy or with an indication for adenotonsillectomy, individuals with rheumatic disorders, individuals with bronchospasm and/or a diagnosis of asthma and/or allergy, individuals with respiratory disorders or important systemic disorders, and individuals undergoing therapies to prevent any recurrent respiratory infections.

\section{Study pattern}

The individuals enrolled following their parents' approval were first subjected to a general medical examination and 
pharyngeal buffer (Test Strep-A; Gima, Gessate, Italy) and then subdivided, according to a simple 1+1 enrolment key, into two groups: one group was treated with Bactoblis ${ }^{\circledR}$, while the other did not receive any treatment and served as the control group. The individuals of the Bactoblis ${ }^{\circledR}$ group were instructed on how to use the product. The product was to be administered for 90 consecutive days. The children had to let it dissolve slowly in the mouth immediately before going to sleep, after brushing their teeth. They were carefully instructed not to chew the product or swallow it whole. They were asked not to drink or swallow any substance following the administration of the product. During the whole trial period, the individuals were invited to come to the clinic as soon as any oropharyngeal symptoms suggested an infection. In this case, the individual was immediately subjected to a medical examination and pharyngeal buffer test. In the case of a positive result, treatment was prescribed to the individual. The prescribed therapy was antibiotics in the case of streptococcal infection. The selected antibiotic in the study was the combination of amoxicillin and clavulanic acid to be administered for 10 days. At the end of the prescribed antibiotic therapy, treatment with Bactoblis ${ }^{\circledR}$ was resumed and continued until the scheduled 90th day. In the case of a viral infection accompanied by pharyngolaryngeal pain and/or a fever, treatment was based on acetaminophen or ibuprofen.

\section{Diagnosis of viral infection}

Diagnosis of viral infection was done according to the following criteria: negative rapid swab for streptococci, absence of submandibular lymphadenopathy, absence of petechiae on the palate, mild dysphagia, absence of headache, absence of abdominal pain, and absence of hyperpyrexia. From a clinical standpoint, patients with viral pharyngitis presented with modest pharyngeal hyperemia, low-grade fever, mild dysphagia, presence of rhinitis with serous secretion, and spontaneous resolution of symptoms without medication in 48-72 hours. Differential diagnosis with mononucleosis, ie, absence of adenomegaly, absence of splenomegaly, absence of plaque exudates on the tonsils, and absence of hyperpyrexia, was also made.

\section{Study objectives}

The study aimed to verify the following parameters: the efficacy of Bactoblis ${ }^{\circledR}$ in the prevention of pharyngotonsillitis from group A S. pyogenes during the study period, the efficacy of Bactoblis ${ }^{\circledR}$ in reducing viral pharyngotonsillar infections in the same period, the onset of side effects or toxicity while the product was being administered, and the compliance of the individuals. Secondary objectives were the collection of information concerning resorting to antibiotic therapy, treatment with antipyretics, working days lost by parents, and days of absence from school (or preschool for children under 6 years of age).

\section{Statistical analysis}

The equivalence of the two treatment groups in terms of sex and age was determined using Fisher's exact test and the two-tailed Wilcoxon-Mann-Whitney test, respectively. The difference between the two treatment groups in terms of number of pharyngotonsillitis episodes was determined using the two-tailed Wilcoxon-Mann-Whitney test. Statistical software used was JMP ${ }^{\circledR} 10$ for Mac OS X (SAS Institute, Cary, NC, USA), and the threshold for statistical significance was $95 \%$. The sample size was determined in the following way. We estimated the prevalence of pharyngotonsillitis episodes in the untreated population to be $90 \%$ of the whole population, while the application of the product could determine a reduction to $50 \%$. For the hypothesis of $95 \%$ specificity and $90 \%$ test power, in consideration of Fisher's exact test, the resulting sample size amounted to 58 units, to be equally divided into the two treatment branches. It was thus decided to include at least 60 individuals to obtain the required statistical effectiveness.

\section{Results}

Sixty children with a diagnosis of recurrent pharyngotonsillitis were enrolled to highlight the preventive role played by slowly dissolving oral tablets $\left(\right.$ Bactoblis $\left.^{\circledR}\right)$ containing a strain of $S$. salivarius K12 (no less than 1 billion CFU/tablet), against $\beta$-hemolytic streptococcal or viral pharyngotonsillitis. Thirty children were treated with one tablet of product a day for 90 consecutive days. The other 30 children served as the control group in the same period. As shown in Table 1, the two groups did not exhibit such important characteristics as to make them significantly different. The statistical

Table I Characteristics* of the children who completed the study

\begin{tabular}{llll}
\hline & Treated & Untreated & P-value \\
\hline Total number & 30 & 30 & \\
Males & 19 & 13 & 0.20 \\
Age $^{\dagger}$ of males & $6.7 \pm 2.5$ & $6.1 \pm 2.8$ & 0.14 \\
Females $_{\text {Age }}^{\dagger}$ of females & 11 & 17 & 0.20 \\
Episodes/child $^{\ddagger}$ & $5.7 \pm 1.9$ & $5.2 \pm 1.8$ & 0.14 \\
\hline
\end{tabular}

Notes: *Nonsignificant differences between groups; ${ }^{\dagger}$ years \pm standard deviation; ffor the quarter considered for enrolment (January 3I-April 30, 20I2). 
equivalence in sex, age, and number of past episodes between the treatment groups was verified using Fisher's exact test $(P=0.20)$, the Wilcoxon-Mann-Whitney test $(P=0.14)$, and the Wilcoxon-Mann-Whitney test $(P=0.70)$, respectively. Table 2 shows the data on the prevalence of streptococcal pharyngotonsillitis episodes (diagnosed with a rapid pharyngeal swab). During the 90-day treatment in the 2013 quarter, the 30 treated children who completed the study were affected by three episodes of streptococcal etiology: pharyngotonsillitis was diagnosed in two children and scarlet fever in a third child. These same 30 children had suffered from 94 episodes of oral streptococcal infection in the same quarter of the previous year. The children in the control group, who had suffered from 90 episodes of oral streptococcal infection in the same quarter of 2012, suffered from 84 episodes in the same quarter of 2013. Prophylaxis with Bactoblis ${ }^{\circledR}$ reduced the incidence of $\beta$-hemolytic streptococcal infections by some 96\% (about 7\% reduction observed in the control group). The statistical analysis also shows that in 2012, the two study groups did not exhibit statistically significant differences in the onset of tonsillitis (Wilcoxon-Mann-Whitney $P=0.16$ ). On the contrary, in 2013 the two groups were found to differ in a statistically significant manner with regard to the onset of tonsillitis (Wilcoxon-Mann-Whitney $P<0.001$ ). The result is that the odds ratio of suffering from tonsillitis following the administration of Bactoblis ${ }^{\circledR}$ was equal to 0.003 , with a $95 \%$ confidence interval amounting to $0.001-0.026$. The assessment of viral oropharyngeal infections was performed only in one of the medical centers involved in the study. For this reason, the data reported in Table 3 refer to only ten children of either group. As the table shows, the ten children of the treated group had had 25 infections of viral etiology in the corresponding quarter of 2012 , with a prevalence of $100 \%$. In the same quarter of 2013, viral infections diagnosed in the same ten children during treatment were only five, with a prevalence of $30 \%$ (two children with two episodes each

Table 2 Episodes of pharyngotonsillitis caused by Streptococcus pyogenes in the two study groups ( $n=30 /$ group)

\begin{tabular}{lllll}
\hline & Treated & A/C & Untreated & A/C \\
\hline January 31-April 30, 2012 & 94 & 3.1 & 90 & 3.0 \\
January 31-April 30, 2013 & $3^{*, \dagger}$ & 0.1 & $84^{\ddagger}$ & 2.8 \\
\% reduction of episodes & 96.79 & & 6.79 & \\
\hline
\end{tabular}

Notes: $* P<0.001$ versus episodes relatable to the same quarter of 2012 and versus episodes relatable to the control group in the same quarter of 2013; tone child with scarlet fever, two children suffering from one pharyngotonsillitis episode each; ¥ 15 children suffering from four pharyngotonsillitis episodes each, ten children suffering from one pharyngotonsillitis episode each and one from scarlet fever, two children suffering from two pharyngotonsillitis episodes each, three children without any episodes.

Abbreviation: A/C, average/child.
Table 3 Rhino-pharyngotonsillar episodes of viral etiopathogenesis in two subgroups of individuals ( $n=10 /$ group)

\begin{tabular}{llllll}
\hline Treatment & $\mathbf{2 0 I 2}$ & A/C & $\begin{array}{l}\mathbf{2 0 I 3} \\
\text { quarter }\end{array}$ & A/C & Delta $\%$ \\
\hline Treated & 25 & 2.5 & $5^{*}$ & 0.5 & 80 \\
Control & 28 & 2.8 & 24 & 2.4 & 14.3 \\
\hline
\end{tabular}

Note: $* P<0.01$ versus the 2012 quarter and versus the control group result relatable to the 2013 quarter.

Abbreviation: $\mathrm{A} / \mathrm{C}$, average/child.

and one with one episode). In the control group, with the assessment being referred again to ten children, the 28 viral infections diagnosed in the quarter of 2012, with a prevalence of $100 \%$, were reduced to 24 in the corresponding quarter of 2013 (prevalence 100\%). Treatment with Bactoblis ${ }^{\circledR}$ reduced the incidence of viral oropharyngeal infections by $80 \%$ (about 14\% reduction observed in the control group). From a statistical perspective, in 2013 we observed a prevalence of viral episodes in the untreated group of $100 \%$, while in the treated one we observed viral infection only in $30 \%$; by the hypothesis of $95 \%$ specificity, this leads to a test power of $70 \%$. Therefore these results are significant at $P<0.01$. The treatment (Table 4), referable only to the 30 children who had received Bactoblis ${ }^{\circledR}$, was well tolerated and without any side effects worth mentioning. Compliance was very good, with only one child complaining of the bad taste of the product. Because of the perceived poor palatability, the individual dropped out of the study immediately on the first enrolment day. As shown in Table 5, the number of days on which the children were treated with antibiotics or antipyretics were 30 and 16, respectively, in the treated group. Antipyretics were administered for 6 days following pain/fever due to a streptococcal infection and for 10 days for the same reasons, but due to a viral infection; this caused the loss of 16 school days (or preschool days, depending on the child's age) and the loss of 16 working days by the children's parents. In the control group, there were instead 900 antibiotic therapy days and 228 antipyretic therapy days, 180 of which owing to streptococcal infections and 48 owing to viral infections. This caused the loss of 228 school (or preschool) days and the loss of 228

Table 4 Tolerability, compliance, and side effects during the 90 day treatment in 31 individuals enrolled in the Bactoblis ${ }^{\circledR}$ group

\begin{tabular}{llll}
\hline & Tolerability & Compliance & Side effects \\
\hline Excellent & $\mathrm{n}=30$ & $\mathrm{n}=30$ & None \\
Good & $\mathrm{n}=0$ & $\mathrm{n}=0$ & None \\
Acceptable & $\mathrm{n}=0$ & $\mathrm{n}=0$ & None \\
Unacceptable & $\mathrm{n}=I^{*}$ & $\mathrm{n}=I^{*}$ & None \\
\hline
\end{tabular}

Note: *Refused to continue on the very first treatment day due to an obvious distaste for the product. 
Table 5 Days under treatment with antibiotics and/or antipyretics or days off preschool/school or working days lost by parents in the study groups ( $n=30 /$ group)

\begin{tabular}{lllll}
\hline Group & Antibiotics & Antipyretics* & School/preschool & Work \\
\hline Treated & 30 & $6+10$ & 16 & 16 \\
Control & 900 & $180+48$ & 228 & 228
\end{tabular}

Note: *The first number indicates the antipyretic administered because of a streptococcal disease, and the second the antipyretic administered because of a viral disease.

working days. In terms of costs (see Table 6), the treated group reported a total expenditure of slightly over $€ 2,600$. The overall expenditure of the control group amounted to approximately $€ 1,530$. In particular, the treated group spent $€ 2,558.25$ to acquire the product Bactoblis ${ }^{\circledR}, € 44.79$ for antibiotics (about $€ 11$ borne by the families and the remainder by the public health service) and $€ 36.80$ to buy antipyretics. The control group spent $€ 1,343.70$ in antibiotics ( $€ 335.70$ borne by the families and the remainder by the public health service) and $€ 184$ in antipyretics.

\section{Discussion}

The chief objective of treatment with probiotics - that is, with strains isolated from human feces (or from the feces of mice, pigs, or other animals) - is the achievement of intestinal health. Probiotics are described as capable of counteracting constipation, diarrhea, or irritable bowel syndrome. These effects can be achieved, but are closely linked to their intestinal colonization capability. Probiotic therapy may be also aimed at a nonintestinal benefit. Examples of these applications are attempts to prevent or treat such gynecological and/or urological conditions as vaginitis, vaginosis, or cystitis, particularly in patients with recurrent forms. Even in these cases, however, the effect seems to be directly connected to the ability of the probiotic to colonize the intestine. Once the strain has reached and colonized the intestine, it can transmigrate to the surrounding, anatomically connected tissues, like the vagina or bladder, and exert its biological action by counteracting the growth of pathogens. ${ }^{18}$ In the case of the K12 strain, the issue of this study, things are different. This strain, isolated from the oral cavity through a pharyngeal swab, can colonize the oropharynx rather than the intestine and locally release the two lantibiotics (salivaricin A2 and B),

Table 6 Expenses $(€)$ borne by the two groups to buy Bactoblis ${ }^{\circledR}$, antibiotics, and antipyretics ( $\mathrm{n}=30 /$ group)

\begin{tabular}{lllll}
\hline Group & Bactoblis $^{\circledR}$ & Antibiotics & Antipyretics & Total \\
\hline Treated & $2,558.25$ & 44.79 & 36.80 & $2,639.84$ \\
Control & 0 & $1,343.70$ & 184.00 & $1,527.70$ \\
\hline
\end{tabular}

which have been described as able to counteract the action of $\beta$-hemolytic streptococcus. Its oral colonization reduces the risk of oral colonization by $S$. pyogenes. As a matter of fact, the same antagonism described for $\beta$-hemolytic streptococcus has also been observed for other strains, which are also sensitive to the same two lantibiotics and responsible for acute otitis media and halitosis. More recent information ${ }^{5}$ seems to demonstrate that oral administration of the K12 strain can not only colonize the oropharynx with the release of the two salivaricins acting as antagonists for certain pathogenic strains but also, through a still not perfectly clear molecular mechanism, reduce IL-8 plasma concentrations and increase salivary interferon- $\gamma$. These modulations may also rationally account for anti-inflammatory and antiviral activity, which would then be added to the antibacterial action of the K12 strain already described. The results of the trial described in this work go exactly in this direction. Prophylaxis with the K12 strain reduced streptococcal infections by over $90 \%$, thus confirming the results of other works, ${ }^{11,12}$ but - and this is something new - also demonstrated an $80 \%$ reduction of viral infections. Use of the K12 strain also contributed to an important reduction in the children's and their parents' days off school and work. In consideration of the need to rest at home during a high-grade fever or high infectivity hazard, use of the K12 strain resulted in absence from school, preschool, or work for 32 days in all, corresponding to about 1 day per family. On the contrary, not using it caused absence from school/preschool/work for 456 days, corresponding to about 15 days per family. With regard to the expenditure parameter, the advantage of not having lost 14 school/working days per family cost the group treated with the K12 strain about $€ 900$ more than the expenditure borne by the control group. To put this in individual family terms, this $€ 900$ delta corresponds to a cost of $€ 30$ per family, and $€ 30$ spread over 90 treatment days corresponds to $33 \mathrm{c}$ a day per family. The same calculation can be demonstrated by arguing on the basis of not losing school days and working days. In this case, as stated earlier, use of the K12 strain prevented the loss of 14 days off school or work per family. As each family spent $€ 30$ more than those who did not resort to the K12 strain, it can be stated that each "saved" day cost about $€ 2$ per family. Hiring a babysitter to avoid losing a working day will certainly cost much more than that. The most important benefit offered by the use of the K12 strain in children suffering from recurrent oral infections of a streptococcal nature is not the undoubted economic advantage, but rather the dramatic reduction in the number of days under antibiotic therapy. As shown in Table 5, in the group treated with 
the K12 strain, resorting to antibiotic therapy was 30 times lower. Similar data can be evinced from those resorting to antipyretic/anti-inflammatory/analgesic therapy. In this case, use of the K12 strain reduced the occurrence of resorting to acetaminophen or ibuprofen 14-fold. These results, together with the excellent tolerability and compliance, as well as the absence of side effects, demonstrate that the K12 strain can be a valid therapeutic solution in the prevention of infective diseases of the oral cavity, whether of streptococcal or viral etiology, and in particular those of a recurrent nature. This study confirms, at least with regard to streptococcal infection prevention, the data observed and published in two previous works, ${ }^{11,12}$ and adds another piece of information: the prevention of viral infections, which will obviously require further clinical confirmation before it can be validated with greater certainty. This study certainly exhibits some limits: the absence of a placebo group, the absence of blind conditions, the small size of the sample, only one center involved in checking viral infection, and an inability to follow up the enrolled children in the 6-9 subsequent months to assess further trends in infective oropharyngeal events. These limits will be overcome in a subsequent multicenter study presently in progress in the Emilia Romagna (Italy) territory.

\section{Disclosure}

FDP is the main formulator of the tested product and involved in the scientific council of the company (Omeopiacenza) trading the tested product. The other authors report no conflicts of interest in this work.

\section{References}

1. Tagg JR. Prevention of streptococcal pharyngitis by anti-Streptococcus pyogenes bacteriocin-like inhibitory substances (BLIS) produced by Streptococcus salivarius. Indian J Med. 2004;119 Suppl:13-16.

2. Hyink O, Wescombe PA, Upton M, Ragland N, Burton JP, Tagg. Salivaricin A2 and the novel lantibiotic salivaricin B are encoded at adjacent loci on a 190-kilobase transmissible megaplasmid in the oral probiotic strain Streptococcus salivarius K12. Appl Environ Microbiol. 2007;73(4):1107-1113.

3. Sharma S, Verma KK. Skin and soft tissue infection. Indian J Pediatr. 2001;68 Supp1 3:S46-S50.
4. Wescombe PA, Burton JP, Cadieux PA, et al. Megaplasmids encode differing combinations of lantibiotics in Streptococcus salivarius. Antonie Van Leeuwenhoek. 2006;90(3):269-280.

5. Wescombe PA, Hale JD, Heng NC, Tagg JR. Developing oral probiotics from Streptococcus salivarius. Future Microbiol. 2012;7(12): $1355-1371$.

6. van Zon A, van der Heijden GJ, van Dongen TM, Burton MJ, Schilder AG. Antibiotics for otitis media with effusion in children. Cochrane Database Syst Rev. 2012;9:CD009163.

7. Power DA, Burton JP, Chilcott CN, Dawes PJ, Tagg JR. Preliminary investigations of the colonisation of upper respiratory tract tissues of infants using a paediatric formulation of the oral probiotic Streptococcus salivarius K12. Eur J Clin Microbiol Infect Dis. 2008;27(12): 1261-1263.

8. Horz HP, Meinelt A, Houben B, Conrads G. Distribution and persistence of probiotic Streptococcus salivarius K12 in the human oral cavity as determined by real-time quantitative polymerase chain reaction. Oral Microbiol Immunol. 2007;22(2):126-130.

9. Burton JP, Wescombe PA, Moore CJ, Chilcott CN, Tagg JR. Safety assessment of the oral cavity probiotic Streptococcus salivarius K12. Appl Environ Microbiol. 2006;72(4):3050-3053.

10. Burton JP, Cowley S, Simon RR, McKinney J, Wescombe PA, Tagg JR. Evaluation of safety and human tolerance of the oral probiotic Streptococcus salivarius K12: a randomized, placebo-controlled, double-blind study. Food Chem Toxicol. 2011;49(9):2356-2364.

11. Di Pierro F, Adami T, Rapacioli G, Giardini N, Streitberger C. Clinical evaluation of the oral probiotic Streptococcus salivarius K12 in the prevention of recurrent pharyngitis and/or tonsillitis caused by Streptococcus pyogenes in adults. Expert Opin Biol Ther. 2013;13(3): 339-343.

12. Di Pierro F, Donato G, Fomia F, et al. Preliminary pediatric clinical evaluation of the oral probiotic Streptococcus salivarius $\mathrm{K} 12$ in preventing recurrent pharyngitis and/or tonsillitis caused by Streptococcus pyogenes and recurrent acute otitis media. Int J Gen Med. 2012;5:991-997.

13. Burton JP, Chilcott CN, Tagg JR. The rationale and potential for the reduction of oral malodour using Streptococcus salivarius probiotics. Oral Dis. 2005;11 Supp1 1:29-31.

14. Burton JP, Chilcott CN, Moore CJ, Speiser G, Tagg JR. A preliminary study of the effect of probiotic Streptococcus salivarius K12 on oral malodour parameters. J Appl Microbiol. 2006;100(4):754-764.

15. Ishijima SA, Hayama K, Burton JP, et al. Effect of Streptococcus salivarius $\mathrm{K} 12$ on the in vitro growth of Candida albicans and its protective effect in an oral candidiasis model. Appl Environ Microbiol. 2012;78(7):2190-2199.

16. Murray RC, Chennupati SK. Chronic streptococcal and non-streptococcal pharyngitis. Infect Disord Drug Targets. 2012;12(4):281-285.

17. Bonsignori F, Chiappini E, De Martino M. The infections of the upper respiratory tract in children. Int J Immunopathol Pharmacol. 2010;23(Suppl 1):16-19.

18. Singh VP, Sharma J, Babu S, Rizwanulla MT, Singla A. Role of probiotics in health and disease: a review. J Pak Med Assoc. 2013;63(2): 253-257.
Drug, Healthcare and Patient Safety

\section{Publish your work in this journal}

Drug, Healthcare and Patient Safety is an international, peer-reviewed open-access journal exploring patient safety issues in the healthcare continuum from diagnostic and screening interventions through to treatment, drug therapy and surgery. The journal is characterized by the rapid reporting of reviews, original research, clinical, epidemiological and

\section{Dovepress}

post-marketing surveillance studies, risk management, health literacy and educational programs across all areas of healthcare delivery. The manuscript management system is completely online and includes a very quick and fair peer-review system. Visit http://www.dovepress.com/ testimonials.php to read real quotes from published authors. 